\title{
Decellularization of Chondrocyte-Encapsulated Collagen Microspheres: A Three-Dimensional Model to Study the Effects of Acellular Matrix on Stem Cell Fate
}

\author{
Hiu-Wa Cheng, B.Eng., ${ }^{1}$ Yuen-Kee Tsui, M.Phil., ${ }^{2}$ Kenneth M.C. Cheung, M.D., ${ }^{2}$ \\ Danny Chan, Ph.D., ${ }^{3}$ and Barbara P. Chan, Ph.D. ${ }^{1}$
}

Extracellular matrix (ECM) partially constitutes the stem cell niche. Reconstituting the ECM niche in a threedimensional (3D) configuration will significantly enhance our understanding of how stem cells interact with and respond to the ECM niche. In this study, we aimed to reconstitute a glycosaminoglycan (GAG)-rich ECM using a microencapsulation technology, produce acellular matrix using a decellularization technique, and investigate the effect of acellular matrix on stem cell fate by repopulating the matrix with human mesenchymal stem cells (hMSCs). We demonstrated that porcine chondrocytes were able to deposit a GAG-rich ECM within the 3D collagen microsphere. All decellularization treatment groups resulted in significant removal of chondrocyte nuclei, but acellular matrix was only achieved using $2 \%$ sodium deoxycholate. Nevertheless, decellularization resulted in significant loss in GAG content in almost all treatment groups, and the $2 \%$ sodium deoxycholate group was able to preserve about $40 \%$ of the GAGs compared with the control group. We further demonstrated that hMSCs seeded onto the decellularized microspheres were able to survive and penetrate into the centre, while hMSCs seeded in the acellular matrix showed positive immunostaining against sox9, indicating that they may be differentiating toward the chondrogenic lineage without the need to supplement the chondrogenic differentiation medium.

\section{Introduction}

$\mathbf{T}$ HE STEM CELL MICROENVIRONMENT or niche is collectively made up of the external signals that control stem cell fate, that is, that either keep them as stem cells or direct them along particular differentiation pathways. ${ }^{1}$ These external control signals include not only the well-known secreted factors and cell-cell interactions but also the extracellular matrix (ECM). ${ }^{1}$ ECM serves as the structural support for stem cells to attach and survive and also controls the stem cell fate by altering the cell-matrix interactions., ${ }^{2,3}$ Moreover, ECM sequesters, modulates, and potentiates secreted factors within the stem cell niche such as binding of bone morphogenetic protein 2 to glycosaminoglycans (GAGs) ${ }^{4}$ and enhancing the basic fibroblast growth factor activities by heparin sulfate proteoglycans. ${ }^{5,6}$ Existing experimental models studying the effect of particular ECM on stem cell fate are usually based on supplementing the free ECM to the culture medium ${ }^{6,7}$ or coating the substratum of the culture chamber with the ECM. ${ }^{8}$ Nevertheless, it has re- cently been demonstrated that cells interact with their ECM differently when they are in a three-dimensional (3D) configuration compared with in $2 \mathrm{D}$ monolayer cultures. ${ }^{9}$ As a result, experimental models reconstituting the ECM microenvironment or niche in a 3D configuration will significantly enhance our understanding of how stem cells interact with and respond to the ECM niche before scaffolding strategies with appropriate controls over the stem cell fates can be developed. ${ }^{10}$

Acellular ECM processed from allogenic or xenogenic tissues is most nature simulating and has been used as scaffolds for the tissue engineering of many tissues, including heart valves, ${ }^{11,12}$ vessels, ${ }^{13}$ nerves, ${ }^{14}$ tendons, and ligaments. ${ }^{15}$ Decellularization is a technique for removing cellular components from native tissues and is usually achieved by a combination of physical, chemical, and enzymatic methods. ${ }^{16-18}$ This technique removes the allogenic or xenogenic cellular antigens from the tissues, as they are the sources for immunogenicity on implantation, but it preserves the ECM components, which are highly conserved among species and

\footnotetext{
${ }^{1}$ Medical Engineering Program, Department of Mechanical Engineering; Departments of ${ }^{2}$ Orthopaedics \& Traumatology and ${ }^{3}$ Biochemistry, Li Ka Shing Faculty of Medicine; The University of Hong Kong, Hong Kong, China.
} 
therefore well-tolerated immunologically. Moreover, growth factors that may facilitate cell growth and remodeling may be preserved in the decellularized matrix. ${ }^{17}$ Decellularized tissues are usually used directly for replacement of defective tissues but are seldom used to investigate the effects of the ECM microenvironment on stem cells. This is because repopulating decellularized native tissues with stem cells is always problematic due to the limited cell penetration and ECM porosity.

Recently, we developed a collagen microencapsulation technique $^{19}$ that entraps living cells in a self-assembled meshwork of native collagen fiber, forming cell-collagen microspheres. The matrix meshwork provides 3D support for cells such as human mesenchymal stem cells (hMSCs) ${ }^{19}$ and embryonic stem cells ${ }^{20}$ to survive, proliferate, migrate, and differentiate. We also demonstrated that stem cells embedded within the meshwork are able to remodel the matrix meshwork by depositing cell-specific ECM when they are exposed to differentiation signals. Examples include GAG production by hMSCs undergoing chondrogenic differentiation $^{21}$ and calcium deposition by hMSCs undergoing osteogenic differentiation. ${ }^{22}$

In this study, we aimed to use the microencapsulation technology together with the decellularization technique to establish a 3D experimental model for investigation of the effects of the cell-specific ECM microenvironment or niche on stem cell fate. Specifically, we aimed to reconstitute a GAGrich $\mathrm{ECM}$ in a $3 \mathrm{D}$ configuration by encapsulating and culturing porcine chondrocytes in the collagen meshwork, produce acellular matrix by decellularizing the chondrocytes with partial retention of the newly deposited matrix, and investigate the effects of the acellular matrix on stem cell fate by repopulating the decellularized matrix with hMSCs.

\section{Materials and Methods}

\section{Culture of primary porcine chondrocytes}

Primary porcine chondrocytes were kindly provided by the Department of Orthopaedics and Traumatology, the University of Hong Kong. Chondrocytes at P0 to P3 were used in this study because chondrocytes of later passages may de-differentiate. Chondrocytes were cultured in full medium consisting of Dulbecco's modified Eagle's mediumhigh glucose (DMEM-HG), 10\% fetal bovine serum (FBS), penicillin $100 \mathrm{U} / \mathrm{mL}$, streptomycin $100 \mathrm{mg} / \mathrm{mL}$, sodium hydrogen carbonate $1.875 \mathrm{mg} / \mathrm{mL}, 0.02 \mathrm{M}$ 4-(2-hydroxyethyl)1-piperazineethanesulfonic acid (HEPES), $1 \mathrm{mM}$ sodium pyruvate, and L-glutamine $0.29 \mathrm{mg} / \mathrm{mL}$. The final $\mathrm{pH}$ of the medium was adjusted to 7.4 with $1 \mathrm{~N}$ sodium hydroxide. Chondrocytes were isolated by adherence selection for $24 \mathrm{~h}$ in cultures. Cells were maintained in full medium with replenishment every 3 days. Chondrocytes at subconfluence were detached by $0.25 \%$ trypsin/ethylenediaminetetraacetic acid (EDTA) for subsequent microencapsulation.

\section{Microencapsulation of chondrocytes}

Chondrocytes were microencapsulated in collagen as previously reported. ${ }^{19}$ In brief, ice-cold rat tail collagen type I (Becton Dickenson, Franklin Lakes, NJ) was neutralized with $1 \mathrm{~N}$ sodium hydroxide and was further diluted with full medium to give a final concentration of $2 \mathrm{mg} / \mathrm{mL}$. Aliquots of chondrocytes at P0 to P3 in full medium were rapidly mixed with the neutralized collagen solution, resulting in cell-matrix mixtures with final cell densities of 500, 2500, and 12,500 cells $/ 2.5 \mu \mathrm{L}$ droplets. Liquid droplets were dispensed into a $35 \mathrm{~mm}$ diameter Petri dish (Sterlin, Stone, United Kingdom) with UV irradiated parafilm covering the substratum. After incubating for $1 \mathrm{~h}$, the liquid droplets gelated to form solid chondrocyte-collagen microspheres, which were then gently flushed into a Petri dish using full medium and cultured for 7 days before decellularization.

\section{Decellularization of chondrocyte-collagen microspheres}

Chondrocyte-collagen microspheres were decellularized by incubation with different detergents at various concentrations, specifically $2 \%$ and $3 \%$ Triton $X-100$, and $0.5 \%, 1 \%$, and $2 \%$ sodium $(\mathrm{Na})$ deoxycholate. For each detergent treatment group, 100 microspheres were immersed in $2 \mathrm{~mL}$ of chemical extraction solutions in $35 \mathrm{~mm}$ diameter Petri dishes for $1 \mathrm{~h}$ at room temperature. Microspheres were rinsed with phosphate-buffered saline (PBS) five times to remove excess detergent before subsequent evaluation and hMSC seeding experiment.

\section{Histological evaluation of chondrocyte-collagen microspheres}

Microspheres were fixed in $4 \%$ paraformaldehyde for $3 \mathrm{~h}$. They were then washed with PBS again to remove excess paraformaldehyde. The fixed microspheres were then embedded in agarose and subsequently stored in 30\% sucrose solution at $4{ }^{\circ} \mathrm{C}$ overnight. The sample was then embedded in optimal cutting temperature (OCT) for cryosectioning. Sections of $10 \mu \mathrm{m}$ thick were prepared for routine hematoxylin and eosin (H\&E) staining to reveal the cell morphology and Alcian blue staining with Nuclear Fast Red counterstains to reveal the GAG-rich region.

\section{Enumeration of cell nuclei in decellularized microspheres}

Cell nuclei were counted in H\&E-stained sections using the image analysis software MetaMorph. The same observer analyzed all the samples in the study in a randomized and blind fashion. Cell densities in the samples were calculated, and the percent reduction in cell density in treated samples (relative to the untreated controls) was determined. In brief, the images were converted to grayscale before analysis for better contrast. An area of $\sim 0.15 \mathrm{~mm}^{2}$, which accounts for more than $95 \%$ of the section, was analyzed. Cell nuclei ranging from 3 to 289 were counted. Four sections with $40 \times$ magnification in the control and each experimental group were analyzed, and the mean was taken as the cell nuclei density (cells $/ \mathrm{mm}^{2}$ ).

\section{Quantification of sulfated GAG content in decellularized microspheres}

For each treatment group and the control group, $10 \mathrm{mi}-$ crospheres per sample were placed in a phosphate buffer at pH 6.5 (50 mM phosphate buffer, $5 \mathrm{mM}$ EDTA, and $5 \mathrm{mM}$ L-cysteine) containing $300 \mu \mathrm{g} / \mathrm{mL}$ papain to solubilize the proteoglycans. Digestion was carried out at $60^{\circ} \mathrm{C}$ overnight. 
A 1,9-dimethylmethylene blue dye-binding assay for sulfated GAGs, using chondroitin sulfate as a standard, was performed on the digested samples. ${ }^{23}$ Absorbance at $656 \mathrm{~nm}$ was then measured using a microplate reader (Safire 2; Tecan, Mannedorf, Switzerland). The quantities of GAGs in samples were determined using a calibration curve prepared by chondroitin sulfate standard with a linear region between 0.125 and $4 \mu \mathrm{g} / 200 \mu \mathrm{L}$.

\section{Culture of hMSCs}

hMSCs were kindly provided by Dr. GCF Chan from the Department of Paediatrics and Adolescent Medicine, the University of Hong Kong. Bone marrow aspirates were collected from healthy bone marrow donors with informed consent. All procedures were approved by the Combined Clinical Ethics Committee of the University of Hong Kong and the Hong Kong West Cluster Hospitals of the Hospital Authority. hMSCs were isolated and cultured as previously reported. ${ }^{24}$ Mononuclear cells were collected after FicollHypaque gradient separation and cultured in full medium consisting of DMEM-low glucose (DMEM-LG) $(1 \mathrm{~g} / \mathrm{L}), 10 \%$ FBS, penicillin $100 \mathrm{U} / \mathrm{mL}$, streptomycin $100 \mathrm{mg} / \mathrm{mL}$, and $2 \mathrm{mM}$ L-glutamine. hMSCs were selected by adherence after $24 \mathrm{~h}$ and maintained in full medium with regular replacement. Cells were detached by $0.05 \%$ trypsin $/ 25$ mmol EDTA and subcultured as P1. hMSCs at P4 were seeded onto the decellularized microspheres.

\section{hMSC seeding on the decellularized microspheres}

The decellularized microspheres were washed with PBS and were equilibrated with the hMSC full medium consisting of DMEM-LG with $10 \%$ FBS for $1 \mathrm{~h}$ before cell seeding. They were then transferred to a parafilm-coated Petri dish without supplementation of medium, and $5 \mu \mathrm{L}$ of hMSC solution at a concentration of $1 \times 10^{6}$ cell $/ \mathrm{mL}$ was directly dropped onto each microsphere. hMSCs were then allowed to adhere to the microspheres for $30 \mathrm{~min}$ before careful supplementation with hMSC medium for further culture.

\section{Cell viability of hMSCs seeded in the microspheres}

hMSCs were cultured for 7, 14, and 21 days before analysis for cell viability and histological and immunohistochemical evaluation. Microspheres were labeled with $2 \mu \mathrm{M}$ calcein AM and $4 \mu \mathrm{M}$ ethidium homodimer-1 for simultaneous staining of live and dead cells, respectively (LIVE/ DEAD Viability/Cytotoxicity kit; Molecular Probes, Invitrogen, Carlsbad, CA). Briefly, the microspheres were washed with PBS to remove all medium, and incubated with the two fluorescent dyes for $45 \mathrm{~min}$ in darkness. The microspheres were subsequently washed again with PBS to remove all labeling medium. Finally, the cell viability of the microspheres was examined using a LEICA DMIRB inverted fluorescent microscope equipped with a SPOT FLEX camera.

\section{Immunohistochemical evaluation of chondrogenic lineages}

Immunohistochemistry against sox 9 was used to reveal the chondrogenic lineage of hMSCs in the seeding experiment. Cryosections of $10 \mu \mathrm{m}$ thickness were incubated with $0.5 \%$ pepsin in $5 \mathrm{mM} \mathrm{HCl}$ at $37^{\circ} \mathrm{C}$ for $30 \mathrm{~min}$ for antigen retrieval. Tissue sections were then treated with $3 \% \mathrm{H}_{2} \mathrm{O}_{2}$ in methanol for $30 \mathrm{~min}$ to block endogenous peroxidase activity. Rabbit anti-sox9 polyclonal antibody (Santa Cruz Biotechnology, Santa Cruz, CA) was used as a primary antibody, diluted to $1: 500$ in $2 \%$ bovine serum albumin in PBS. Sections were incubated with primary antibody at $4{ }^{\circ} \mathrm{C}$ overnight before incubation with the secondary antibody, the anti-rabbit immunoglobulin, which was diluted to 1:2000 in $2 \%$ bovine serum albumin in PBS, for $30 \mathrm{~min}$ at room temperature. The Vectastain ABC kit (Vector Laboratories, Burlingame, CA) was used to observe the positive signal, and hematoxylin was used as the counterstain.

\section{Data presentation and statistical analysis}

Quantitative results such as cell nucleus density and quantity of GAGs were reported as mean \pm standard deviation. One-way ANOVA with appropriate post hoc tests were used to reveal differences among different treatment groups. A significance level was set at 0.05 , and SPSS 16.0 was used for the analysis.

\section{Results}

\section{Characterization of chondrocyte-encapsulated microspheres}

Figure 1 shows the morphological, histological, histochemical, and immunohistochemical characterization of chondrocyteencapsulated microspheres. Phase contrast microscopy showed that porcine chondrocytes were entrapped in 3D collagen microspheres. H\&E staining showed chondrocytes resided in lacunae in the collagen matrix of the microspheres. Alcian blue staining showed that chondrocytes were able to deposit GAGs in the ECM of the microspheres. Immunohistochemical staining of $\operatorname{sox} 9$ in nuclei confirmed the identity of the chondrocytes.

\section{Removal of cell nuclei from chondrocyte-entrapped microspheres}

Figure 2 shows the histological staining of chondrocyteentrapped collagen microspheres in different treatment groups. Chondrocytes, stained blue in H\&E staining, were randomly distributed in collagen microspheres without decellularization treatment (Fig. 2A). Chondrocyte-entrapped collagen microspheres treated with Triton X-100 at different concentrations (Fig. 2B, C) showed dramatically reduced cell density compared with the control group, but cell nuclei and cytoplasm were still distinct. As for Na deoxycholate, $0.5 \%$ and $1 \%$ also significantly reduced the cell density compared with the control group, but again cell nuclei and cytoplasm were still distinct. Increasing the concentration to $2.0 \%$ successfully removed all cell nuclei in the microspheres, although some cytoplasm was still visible. The number of nuclei in different treatment groups is shown in Figure 3. Treatments with Triton X-100 and lower concentration of $\mathrm{Na}$ deoxycholate resulted in at least a $50 \%$ reduction in the number of nuclei per unit area in the microspheres, while there were less than 15 cells per unit area in the $2 \% \mathrm{Na}$ deoxycholate group. One-way ANOVA showed significant difference among all groups $(p<0.001)$, while post hoc tests showed that all treatment groups were significantly different from the control groups $(p<0.05)$. 
FIG. 1. Microscopic characterization of the chondrocytecollagen microspheres. (A) Phase contrast microscopy; (B) H\&E staining; (C) Alcian blue staining; (D) immunohistochemistry of sox9. Color images available online at www.liebertonline.com/ten.

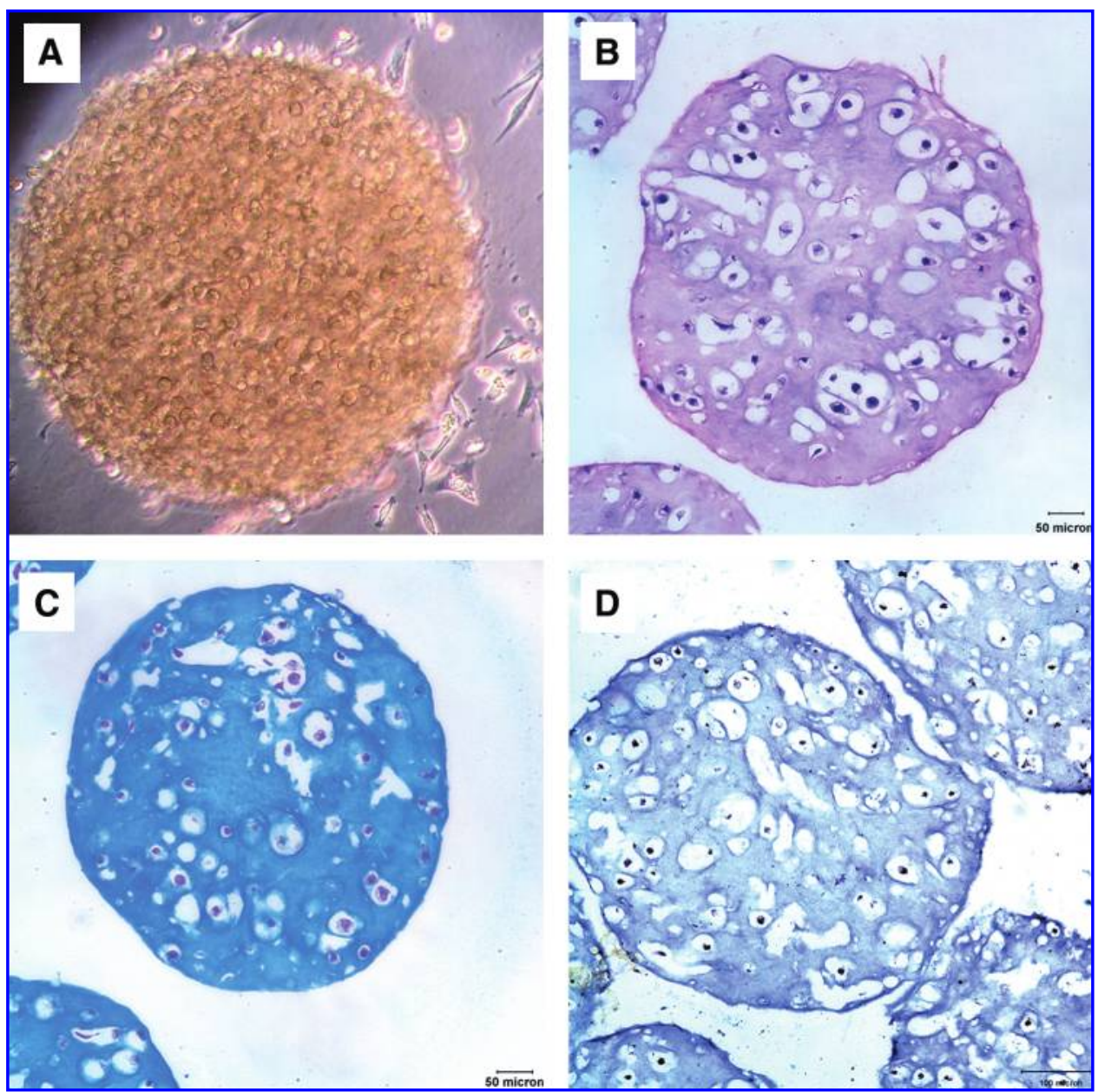

\section{Cell viability of chondrocyte-entrapped microspheres}

Figure 4 shows the cell viability in chondrocyte-entrapped microspheres. In microspheres without any chemical treatment (Fig. 4A), the majority of cells were alive (green cytoplasm), while only a few dead cells (red nuclei) were found. In microspheres treated with $2 \%$ Triton X-100 (Fig. 4B), all cells were dead, and only red nuclei could be located. In microspheres treated with $2 \%$ Na deoxycholate (Fig. 4C), no live cells remained, and no nuclei could be located due to their complete removal.

\section{Preservation of GAG content in chondrocyte-entrapped microspheres}

Figure 5 shows the representative Alcian blue staining for GAG-rich regions and the amount of GAGs in microspheres of different treatment groups. About $2 \%$ of Triton X-100 was able to preserve at least $90 \%$ of GAGs in microspheres compared with the control group, while increasing the concentration to $3 \%$ reduced the GAG content to about only $65 \%$ of the control. For $\mathrm{Na}$ deoxycholate, low concentration at $0.5 \%$ was able to preserve more than $90 \%$ of the GAG content in the microsphere and increasing it to $1 \%$ also preserved more than $80 \%$ of the GAGs. However, $2 \%$ Na deoxycholate only retained about $40 \%$ of the GAGs compared with the control group. One-way ANOVA showed statistically significant difference among different groups $(p<0.001)$, while post hoc tests showed that all groups except the $2 \%$ Triton $\mathrm{X}-100$ group had statistically significant loss of GAGs $(p<0.05)$ compared with the control group.

\section{Morphology of hMSC-seeded decellularized microspheres}

Figure 6 shows the morphology of hMSC-seeded microspheres, which have been decellularized by different chemical treatments. hMSCs were able to grow and penetrate into the center of the decellularized microspheres in both the Triton X-100 and Na deoxycholate groups. Moreover, the cellularity continuously increased over the 3 weeks of culture time in both groups.

\section{Cell viability of hMSCs seeded on decellularized microspheres}

Figure 7 shows the cell viability staining for hMSCs seeded on the decellularized microspheres. Both $2 \%$ Triton X-100 and 2\% Na deoxycholate-treated microspheres were able to support cell survival, and most hMSCs were found viable after culture for 7 days. 

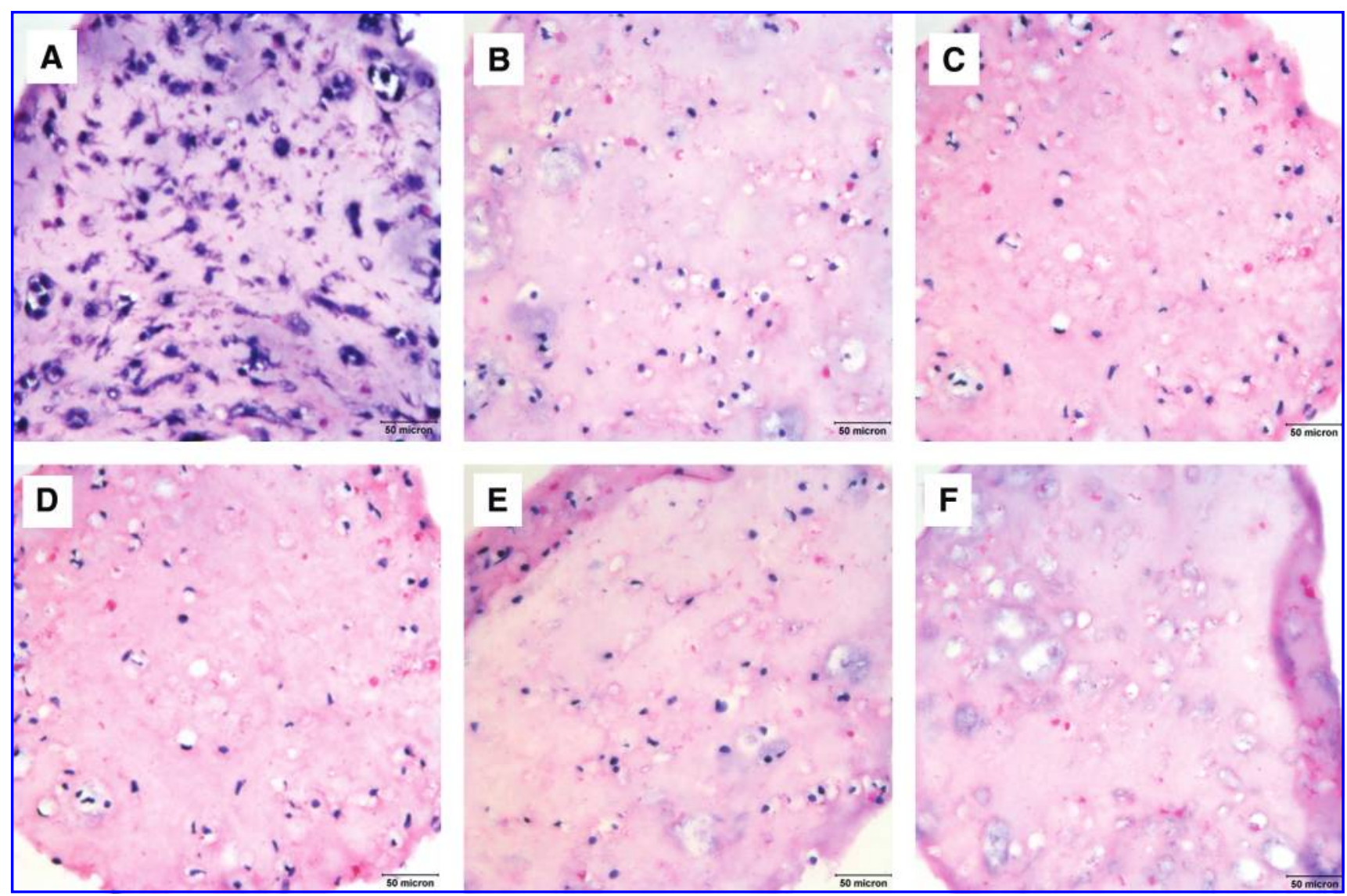

FIG. 2. Representative H\&E staining of the chondrocyte-collagen microspheres in different decellularization treatment groups. (A) Control samples of collagen embedded with chondrocytes; (B) samples treated with 2\% Triton X-100; (C) samples treated with 3\% Triton X-100; (D) samples treated with $0.5 \%$ Na deoxycholate; (E) samples treated with $1 \%$ Na deoxycholate; (F) samples treated with $2 \%$ Na deoxycholate. Color images available online at www.liebertonline.com/ten.

\section{Sox9 immunohistochemistry of hMSC-seeded decellularized microspheres}

Figure 8 shows the immunohistochemical staining for sox 9 in hMSC seeded on the decellularized microspheres with acellular matrix. hMSCs encapsulated in collagen gel without exposure to chondrogenic differentiation signals showed negative staining for sox9, while hMSCs seeded in the acellular matrix in the microspheres produced by decellulariza-

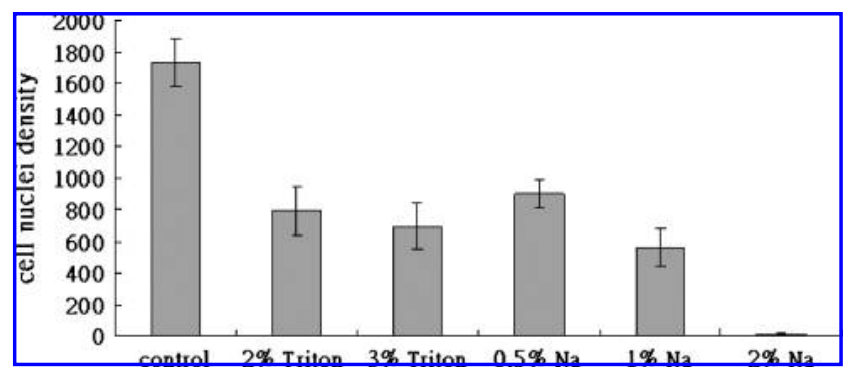

FIG. 3. Bar chart showing the cell nuclei per unit area in chondrocyte-collagen microspheres in different decellularization treatment groups. Data represent mean \pm standard deviation for $n=4$. tion with $2 \% \mathrm{Na}$ deoxycholate showed positive staining in cell nuclei after 7, 14, and 21 days of culture.

\section{Alcian blue staining of hMSC-seeded decellularized microspheres}

Figure 9 shows the Alcian blue staining of the hMSCseeded decellularized microspheres at 7, 14, and 21 days. Increased cellularity with large cell clusters was found in these microspheres in later time points at 14 and 21 days, suggesting that the seeded hMSC could proliferate and grow. Moreover, the seeded cells remodeled the microsphere matrix by digesting away the old GAG-rich matrix, as shown by the reduced proportion of Alcian blue-stained regions $\left(^{*}\right)$ in particular on days 14 and 21. In the mean time, Alcian blue-stained regions could be located within the region with newly proliferated cell clusters (arrows).

\section{Discussion}

This study demonstrates the possibility of producing acellular matrix by first encapsulating and culturing chondrocytes in a template collagen fibrous meshwork and then completely removing the chondrocytes with partial retention of the newly deposited matrix. This study also demonstrates successful repopulation of the acellular matrix with hMSCs 
FIG. 4. Live and dead staining of chondrocytecollagen microspheres in different decellularization treatment groups. (A) Control; (B) $2 \%$ Triton $\mathrm{X}-100$; (C) $2 \% \mathrm{Na}$ deoxycholate. Color images available online at www.liebertonline .com/ten.

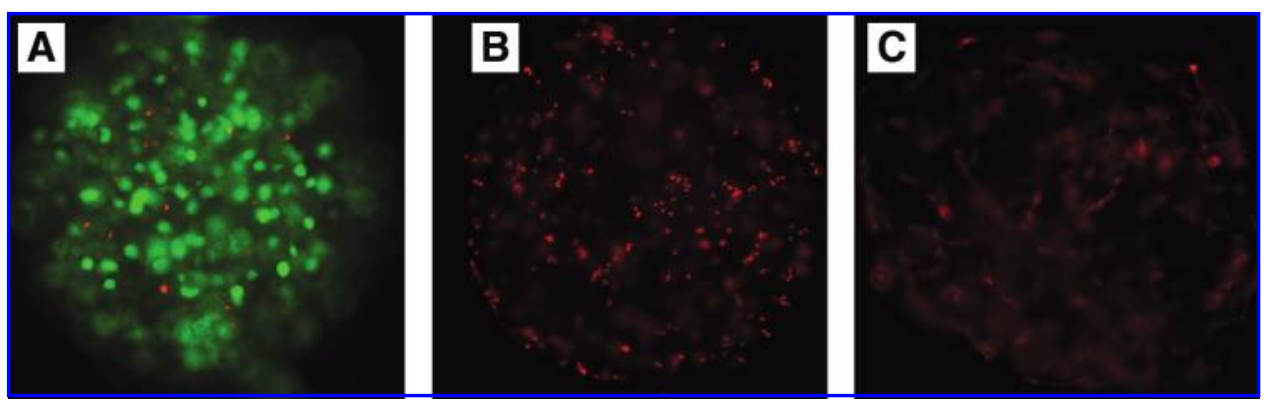

and provides evidence of the commitment of hMSCs repopulated on the acellular matrix toward chondrogenic lineages.

\section{Collagen meshwork acts as the template for encapsulated cells to deposit new matrices}

Collagen microspheres provide 3D fiber meshwork, ${ }^{19}$ which serves as the template to allow cells to remodel and lay down new ECM within the meshwork. ${ }^{21,22}$ This study showed that chondrocytes were able to make a large amount of GAGs within the collagen meshwork. Moreover, our previous reports showed that hMSCs undergoing chondrogenic differentiation laid down GAGs and type II collagen, ${ }^{21}$ while hMSCs undergoing osteogenic differentiation laid down calcium deposits ${ }^{22}$ within the template collagen meshwork in the microspheres. As a result, this microencapsula- tion technique provides a 3D template for various types of cells to reconstitute their specific matrices and can thus be used to study the effects of such a cell-specific matrix microenvironment on the fate of stem cells.

\section{$\mathrm{Na}$ deoxycholate is a better decellularization chemical than Triton $X-100$}

Complete decellularization is essential to avoid the immunogenicity elicited by the cellular remnants. Na deoxycholate is apparently a better decellularization chemical than Triton X-100. The amount of cells removed by $\mathrm{Na}$ deoxycholate is extremely sensitive to the dosage of this chemical, and increasing the concentration to $2 \%$ effectively removed all cell nuclei, although the newly deposited GAGs were only partially retained. Further optimization is required to retain more GAGs by increasing the initial chondrocytes
FIG. 5. Alcian blue staining and quantification of glycosaminoglycans (GAGs) in chondrocytecollagen microspheres of different decellularization treatment groups; (A) control; (B) $2 \% \mathrm{Na}$ deoxycholate group; $(\mathbf{C})$ bar chart showing the amounts of GAGs in microspheres of different treatment groups. Data represent mean \pm standard deviation for $n=7$. Color images available online at www.liebertonline .com/ten.

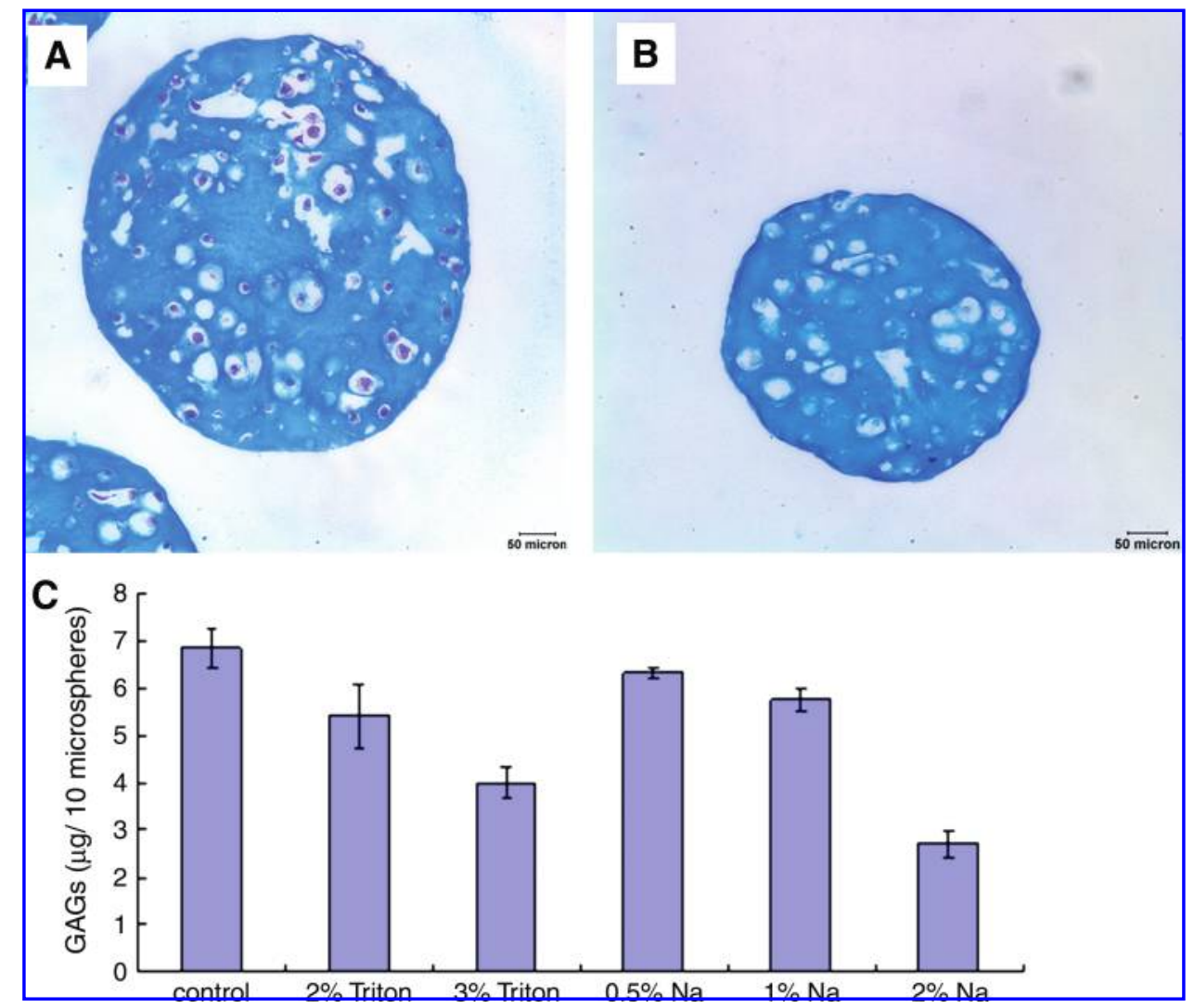




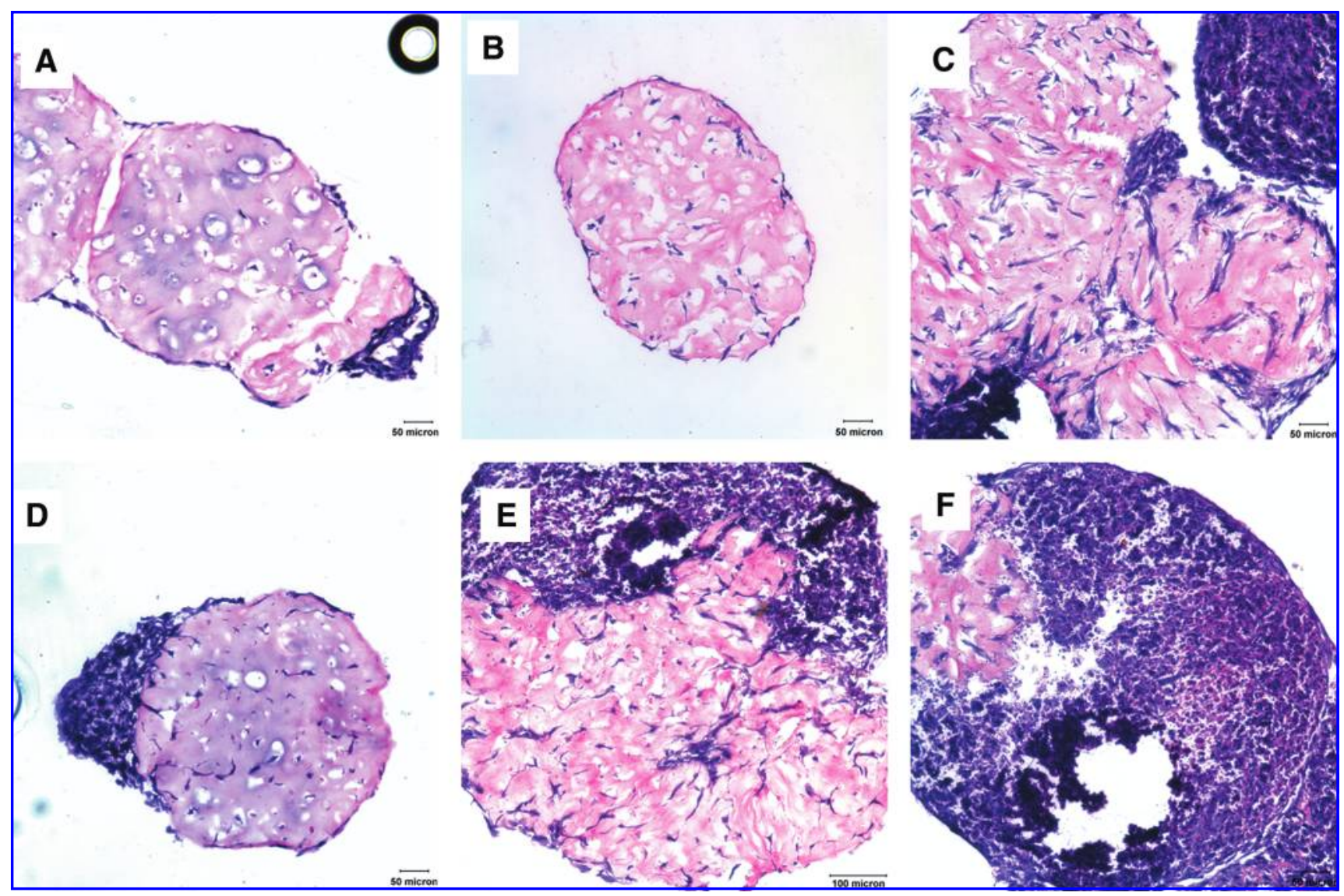

FIG. 6. Representative H\&E staining of human mesenchymal stem cells (hMSCs) seeded on decellularized microspheres. (A-C) hMSCs seeded on 2\% Triton X-100-treated microspheres for 7, 14, and 21 days, respectively; (D-F) hMSCs seeded on $2 \% \mathrm{Na}$ deoxycholate-treated microspheres for 7, 14, and 21 days, respectively. Color images available online at www .liebertonline.com/ten.

seeding density in the microspheres, lengthening the culture period of chondrocytes on the microspheres, or using other extraction chemicals and protocols. Higher concentration at $4 \%$ was not used because it dissolved all microspheres (data not shown). On the other hand, the amount of cells removed is less sensitive to Triton $\mathrm{X}-100$ treatment as concentration lower than $2 \%$ did not significantly remove cells (data not shown), $2 \%$ removed only half of the nuclei, and increasing concentration to 3\% did not further increase the amount of cells removed. Compared with native tissues, which have intact structures other than collagen to provide further mechanical support and resistance to chemical treatment, these collagen microspheres need milder decellularization protocols and shorter decellularization time because proteoglycans are highly water soluble and are easily lost into the surrounding solutions. ${ }^{25}$

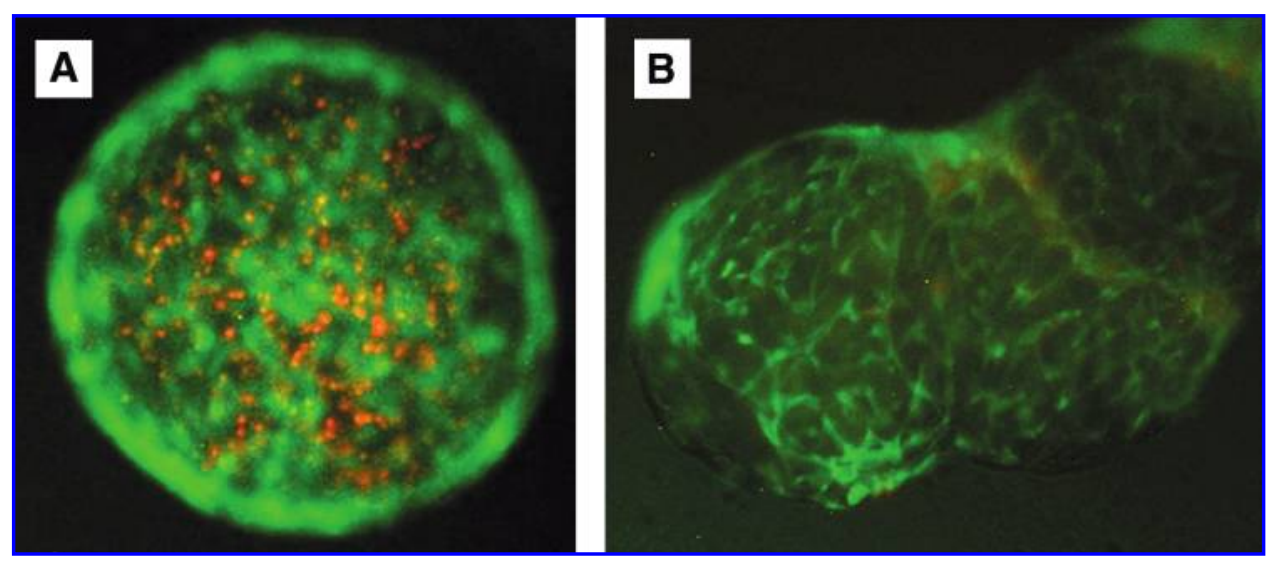

FIG. 7. Live and dead staining of decellularized chondrocyte-collagen microspheres repopulated with hMSCs. (A) Microspheres decellularized by $2 \%$ Triton X-100 showed both live hMSCs (green) and remnant chondrocyte nuclei (red); (B) Microspheres decellularized by $2 \% \mathrm{Na}$ deoxycholate showed only live hMSCs (green). Color images available online at www .liebertonline.com/ten. 


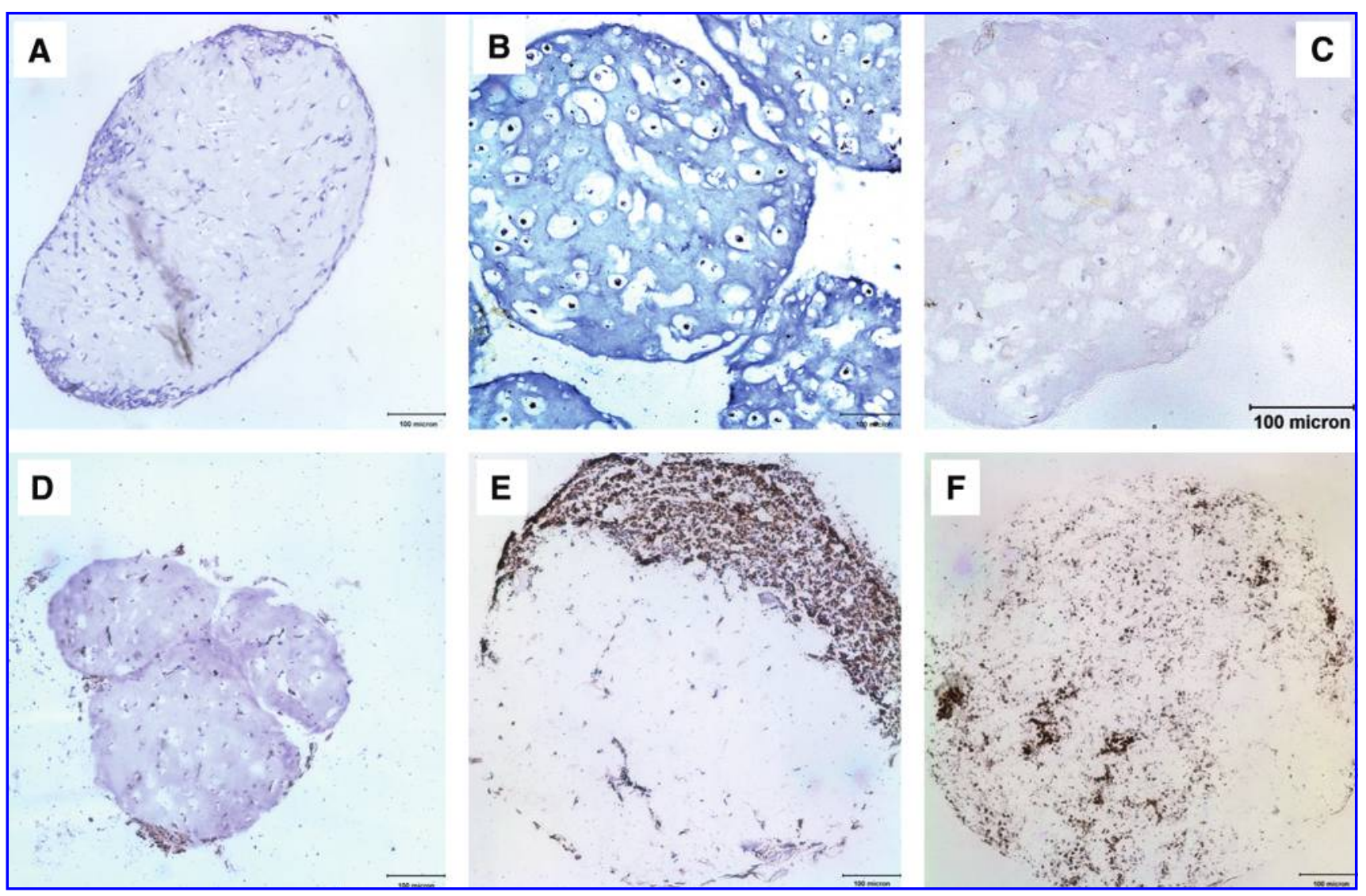

FIG. 8. Immunohistochemistry of sox 9 of hMSCs seeded on decellularized microspheres. (A) Negative control using hMSCcollagen gel cultured for 7 days; (B) positive control using chondrocyte-collagen microspheres cultured for 7 days; (C) negative staining in $2 \% \mathrm{Na}$ deoxycholate decellularized microspheres (no hMSC seeding) for 7 days; (D-F) positive staining in hMSC-seeded 2\% Na deoxycholate decellularized microspheres for 7, 14, and 21 days, respectively. Color images available online at www.liebertonline.com/ten.

Fate of hMSCs seeded in the decellularized microspheres

hMSCs are able to penetrate into the center of the decellularized microspheres and repopulate the whole microsphere, suggesting that even though there is a minute amount of residual decellularization chemical, it does not induce adverse effects on the seeded hMSCs. Positive staining of sox 9 , the intranuclear marker for cells committing to chondrogenic lineage, has been found in hMSCs seeded on microspheres decellularized by $\mathrm{Na}$ deoxycholate for 7, 14, and 21 days, indicating that the hMSCs may be differentiating toward the chondrogenic lineage in the acellular matrix without the need to supplement the chondrogenic
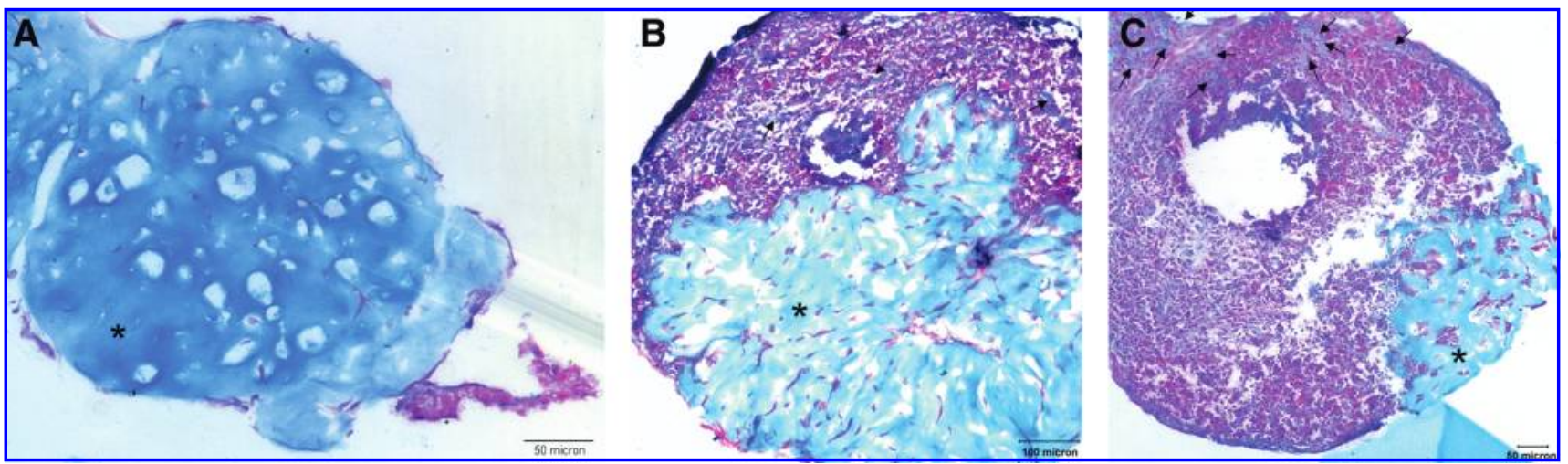

FIG. 9. Representative Alcian blue staining of hMSCs seeded on $2 \% \mathrm{Na}$ deoxycholate decellularized microspheres. hMSCs seeded for 7 (A), 14 (B), and 21 (C) days. Color images available online at www.liebertonline.com/ten. 
differentiation medium. Only Na deoxycholate-treated microspheres were used in this part of the study because this treatment completely removed all cell nuclei of porcine chondrocytes and successfully produced an acellular matrix. As a result, any positive sox 9 staining would be attributable to the newly seeded hMSCs. ECM markers for chondrogenic lineages such as type II collagen and proteoglycans were not used in this study because it is impossible to distinguish the newly deposited ECM by the differentiating hMSCs from the residual ECM components synthesized by the porcine chondrocytes before decellularization. Commitment of hMSCs to chondrogenic lineage may be due to the ECM microenvironment deposited by the porcine chondrocytes and possibly remnant growth factors in the matrix. Previous studies with a similar approach using decellularized matrix showed that osteoblast-specific matrix induced MSCs to commit to the osteogenic lineage. ${ }^{26,27}$ Corroborating with these studies, our results suggest the importance of reconstituting a 3D cell-specific acellular matrix environment to regulate stem cell fate and contribute to further development of biomimetic scaffolding approaches.

\section{Conclusion}

Porcine chondrocytes microencapsulated in collagen microspheres were able to deposit a GAG-rich ECM microenvironment. An optimized decellularization protocol has been established to completely remove the encapsulated chondrocytes with partial retention of the GAG-rich matrix. The decellularized microspheres were able to be repopulated with hMSCs, and the commitment of hMSCs to chondrogenic lineage in the acellular matrix has been demonstrated. This study provides an excellent 3D model to study the cellspecific acellular matrix microenvironment or niche and the effects on stem cells.

\section{Acknowledgments}

This work was supported by grants from AOSpine (AOSBRC-07-06); the Innovation and Technology Commission, the Hong Kong Government (GHP/050/06); the University Research Committee, the University of Hong Kong (10206799); and the Strategic Research Theme on Development, Growth and Reproduction. The authors thank Ms. Vicki Geall for editing the manuscript.

\section{Disclosure Statement}

No competing financial interests exist.

\section{References}

1. Watt, F.M., and Hogan, B.L. Out of Eden: stem cells and their niches. Science 287, 1427, 2000.

2. Jensen, U.B., Lowell, S., and Watt, F.M. The spatial relationship between stem cells and their progeny in the basal layer of human epidermis: a new view based on whole mount labeling and lineage analysis. Development 126, 2409, 1999.

3. Quesenberry, P.J., Becker, P., and Stewart, F.M. Phenotype of the engrafting stem cell in mice. Stem Cells 16, 33, 1998.

4. Grünert, M., Dombrowski, C., Sadasivam, M., Manton, K., Cool, S.M., and Nurcombe, V. Isolation of a native osteoblast matrix with a specific affinity for BMP2. J Mol Histol 38, 393, 2007.

5. Fannon, M., Forsten, K.E., and Nugent, M.A. Potentiation and inhibition of bFGF binding by heparin: a model for regulation of cellular response. Biochemistry 39, 1434, 2000.

6. Rider, D.A., Dombrowski, C., Sawyer, A.A., Ng, G.H., Leong, D., Hutmacher, D.W., Nurcombe, V., and Cool, S.M. Autocrine fibroblast growth factor 2 increases the multipotentiality of human adipose-derived mesenchymal stem cells. Stem Cells 26, 1598, 2008.

7. Sirko, S., von Holst, A., Wizenmann, A., Gotz, M., and Faissner, A. Chondroitin sulfate glycosaminoglycans control proliferation, radial glia cell differentiation and neurogenesis in neural stem/progenitor cells. Development 134, 2727, 2007.

8. Zhu, X., Eibl, O., Scheideler, L., and Geis-Gerstorfer, J. Characterization of nano hydroxyapatite/collagen surfaces and cellular behaviors. J Biomed Mater Res A 79, 114, 2006.

9. Zamir, E., and Geiger, B. Molecular complexity and dynamics of cell-matrix adhesions. I Cell Sci 114, 3583, 2001.

10. Metallo, C.M., Mohr, J.C., Detzel, C.J., de Pablo, J.J., van Wie, B.J., and Palecek, S.P. Engineering the stem cell microenvironment. Biotechnol Prog 23, 18, 2007.

11. Grauss, R.W., Hazekamp, M.G., Oppenhuizen, F., van Munsteren, C.J., Gittenberger-de Groot, A.C., and DeRuiter, M.C. Histological evaluation of decellularised porcine aortic valves: matrix changes due to different decellularisation methods. Eur J Cardiothorac Surg 27, 566, 2005.

12. Oswal, D., Korossis, S., Mirsadraee, S., Wilcox, H., Watterson, K., Fisher, J., and Ingham, E. Biomechanical characterization of decellularized and cross-linked bovine pericardium. J Heart Valve Dis 16, 165, 2007.

13. Borschel, G.H., Huang, Y.C., Calve, S., Arruda, E.M., Lynch, J.B., Dow, D.E., Kuzon, W.M., Dennis, R.G., and Brown, D.L. Tissue engineering of recellularized small-diameter vascular grafts. Tissue Eng 11, 778, 2005.

14. Hall, S. Axonal regeneration through acellular muscle grafts. J Anat 190, 57, 1997.

15. Stapleton, T.W., Ingram, J., Katta, J., Knight, R., Korossis, S., Fisher, J., and Ingham, E. Development and characterization of an acellular porcine medial meniscus for use in tissue engineering. Tissue Eng Part A 14, 505, 2008.

16. Badylak, S.F. The extracellular matrix as a scaffold for tissue reconstruction. Semin Cell Dev Biol 13, 377, 2002.

17. Li, F., Li, W., Johnson, S., Ingram, D., Yoder, M., and Badylak, S. Low-molecular-weight peptides derived from extracellular matrix as chemoattractants for primary endothelial cells. Endothelium 11, 199, 2004.

18. Gilbert, T.W., Sellaro, T.L., and Badylak, S.F. Decellularization of tissues and organs. Biomaterials 27, 3675, 2006.

19. Chan, B.P., Hui, T.Y., Yeung, C.W., Li, J., Mo, I., and Chan, G.C. Self-assembled collagen-human mesenchymal stem cell microspheres for regenerative medicine. Biomaterials 28, 4652, 2007.

20. Yeung, C.W., Cheah, K.S.E., Chan, D., and Chan, B.P. A novel 3-dimensional culture system for mouse embryonic stem cells. International Society for Stem Cell Research 5th Annual Meeting, June 15-21, 2007. Cairns, Queensland, Australia, 2007, p. 340.

21. Hui, T.Y., Cheung, K.M., Cheung, W.L., Chan, D., and Chan, B.P. In vitro chondrogenic differentiation of human mesenchymal stem cells in collagen microspheres: influence of cell seeding density and collagen concentration. Biomaterials 29, 3201, 2008. 
22. Chan, B.P., Hui, T.Y., Wong, M.Y., Yip, H.K., and Chan, G.C.F. Fabrication of osteoinductive bone-like microparticles using mesenchymal stem cell encapsulated collagen microspheres. (Under review.).

23. Barbosa, I., Garcia, S., Barbier-Chassefiere, V., Caruelle, J.P., Martelly, I., and Papy-García, D. Improved and simple micro assay for sulfated glycosaminoglycans quantification in biological extracts and its use in skin and muscle tissue studies. Glycobiology 13, 647, 2003.

24. Li, J., Law, H.K., Lau, Y.L., and Chan, G.C. Differential damage and recovery of human mesenchymal stem cells after exposure to chemotherapeutic agents. Br J Haematol 127, 326, 2004.

25. Roughley, P.J., Alini, M., and Antoniou, J. The role of proteoglycans in aging, degeneration and repair of the intervertebral disc. Biochem Soc Trans 30, 869, 2002.

26. Datta, N., Pham, Q.P., Sharma, U., Sikavitsas, V.I., Jansen, J.A., and Mikos, A.G. In vitro generated extracellular matrix and fluid shear stress synergistically enhance 3D osteoblastic differentiation. Proc Natl Acad Sci USA 103, 2488, 2006.

27. Pham, Q.P., Kasper, F.K., Scott Baggett, L., Raphael, R.M., Jansen, J.A., and Mikos, A.G. The influence of an in vitro generated bone-like extracellular matrix on osteoblastic gene expression of marrow stromal cells. Biomaterials 29, 2729, 2008.

Address correspondence to: Barbara P. Chan, Ph.D. Medical Engineering Program Department of Mechanical Engineering Room 711, Haking Wong Building Pokfulam Road

The University of Hong Kong Hong Kong Special Administrative Region Hong Kong 852

China

E-mail: bpchan@hkucc.hku.hk

Received: November 18, 2008 Accepted: March 4, 2009

Online Publication Date: May 5, 2009 


\section{This article has been cited by:}

1. Takashi Hoshiba, Hongxu Lu, Tomoe Yamada, Naoki Kawazoe, Tetsuya Tateishi, Guoping Chen. 2011. Effects of extracellular matrices derived from different cell sources on chondrocyte functions. Biotechnology Progress 27:3, 788-795. [CrossRef]

2. Chun-hei Li, Tsz-Kit Chik, Alfonso H.W. Ngan, Stanley C.H. Chan, Daisy K.Y. Shum, Barbara P. Chan . 2011. Correlation Between Compositional and Mechanical Properties of Human Mesenchymal Stem Cell-Collagen Microspheres During Chondrogenic DifferentiationCorrelation Between Compositional and Mechanical Properties of Human Mesenchymal Stem Cell-Collagen Microspheres During Chondrogenic Differentiation. Tissue Engineering Part A 17:5-6, 777-788. [Abstract] [Full Text] [PDF] [PDF Plus]

3. D. Adam Young, Dina O. Ibrahim, Diane Hu, Karen L. Christman. 2011. Injectable hydrogel scaffold from decellularized human lipoaspirate. Acta Biomaterialia 7:3, 1040-1049. [CrossRef] 